\title{
Glyoxalase 1 as a candidate for indicating the metastatic potential of SN12C human renal cell carcinoma cell clones
}

\author{
TOSHIYUKI TANAKA ${ }^{1}$, YASUHIRO KURAMITSU ${ }^{1}$, YUFENG WANG $^{1}$, BYRON BARON $^{1}$, \\ TAKAO KITAGAWA ${ }^{1}$, KAZUHIRO TOKUDA ${ }^{1}$, KOSEI HIRAKAWA ${ }^{2}$, MASAKAZU YASHIRO ${ }^{2}$, \\ SEIJI NAITO $^{3}$ and KAZUYUKI NAKAMURA ${ }^{1}$ \\ ${ }^{1}$ Department of Biochemistry and Functional Proteomics, Yamaguchi University Graduate School of Medicine, Ube, \\ Yamaguchi 755-8505; ${ }^{2}$ Department of Surgical Oncology, Osaka City University, Graduate School of Medicine, \\ Osaka $545-8585 ;{ }^{3}$ Department of Urology, Graduate School of Medical Sciences \\ Kyushu University, Fukuoka 812-8582, Japan
}

Received June 20, 2013; Accepted July 16, 2013

DOI: $10.3892 / o r .2013 .2699$

\begin{abstract}
Three clones with differential metastatic potential were established from the parental SN12C human renal cell carcinoma (HRCC). We previously reported that in the two high metastatic SN12C clones, two isoforms of ubiquitin carboxyl-terminal hydrolase isozyme L1 (UCH-L1) showed decreased expression by using two-dimensional electrophoresis (2-DE) covering a $\mathrm{pH}$ range $(\mathrm{pH} 3.0-10.0)$ followed by liquid chromatography-tandem mass spectrometry. However, in the case of the low metastatic clone, the spot volume for UCH-L1 was almost the same as for the parental SN12C. In the present study, we found one protein spot which was correlated with the metastatic potential of SN12C clones by using 2-DE over a narrow $\mathrm{pH}$ range ( $\mathrm{pH} 4.0-7.0)$. The protein glyoxalase 1 (GLO1) appeared to be directly proportional to the metastatic potential of the SN12C clones. GLO1 was the only protein which consistently varied according to the metastatic potentials of SN12C clones. GLO1 was increased in high metastatic cell lines by western blot analysis. These findings suggest that GLO1 is associated with the metastatic potential of SN12C HRCC clones. We expanded our experi-
\end{abstract}

Correspondence to: Dr Yasuhiro Kuramitsu, Department of Biochemistry and Functional Proteomics, Yamaguchi University Graduate School of Medicine, 1-1-1 Minami-kogushi, Ube, Yamaguchi 755-8505, Japan

E-mail: climates@yamaguchi-u.ac.jp

Abbreviations: HRCC, human renal cell carcinoma; 2-DE, two-dimensional electrophoresis; MS, mass spectrometry; LC-MS/MS, liquid chromatography-tandem mass spectrometry; UCH-L1, ubiquitin carboxyl-terminal hydrolase isozyme L1; GLO1, glyoxalase 1; \%SPI, \% scored peak intensity; MG, methylglyoxal; NO, nitric-oxide

Key words: human renal cell carcinoma, metastatic cancer, proteomics, two-dimensional gel electrophoresis mental range to include clones of scirrhous gastric cancer cell lines (OCUM-2M, OCUM-2D and OCUM-2MLN) and similar results were obtained, thereby further strengthening our original findings.

\section{Introduction}

Cancer is a major cause of mortality worldwide, mainly due to metastasis (World Cancer Report 2008; URL: http://www. iarc.fr/en/publications/pdfs-online/wcr/index.php). However, the molecular mechanism of metastasis is not well known. The metastatic cascade has a series of discrete steps, including epithelial-mesenchymal transition, invasion, anoikis, angiogenesis, transport through vessels and outgrowth of secondary tumors. Furthermore, recent findings suggested yet another step, a premetastatic niche, before metastasis becomes evident (1). This cascade is a complex process.

SN12C clones have been established to be a suitable model for spontaneous metastasis (2). SN12C was established from a human renal cell carcinoma (HRCC) surgical specimen (3). SN12C-clone 2 and SN12C-PM6 have higher metastatic potential than parental SN12C, with SN12C-PM6 having the highest metastatic potential of all cell clones $(4,5)$. On the other hand, SN12C-clone 4 has lower metastatic potential than parental SN12C (4).

Our previous studies using two-dimensional electrophoresis (2-DE) covering $\mathrm{pH}$ 3.0-10.0 suggested eight protein spots as potential candidates for high metastatic potential in SN12C cell clones. In particular, ubiquitin carboxyl-terminal hydrolase isozyme L1 (UCH-L1) correlated with high metastatic potential SN12C clones. However, we were not able to show any candidate for the low metastatic potential of SN12C-clone 4 (6). In the present study, we performed proteomic differential display analysis for the four SN12C cell clones with different metastatic potential (3-5) by using 2-DE over a narrow $\mathrm{pH}$ range $(\mathrm{pH} 4.0-7.0)$, since in our previous study, several protein spots were separated in the range of $\mathrm{pH}$ 4.0-7.0 on the gels. In the present study, we showed that glyoxalase 1 (GLO1) increases in high metastatic clones at the 
protein level and we also discussed the biological significance of the increase in the metastatic potential of SN12C HRCC clones and scirrhous gastric cancer OCUM.

\section{Materials and methods}

Tumor cell lines and culture conditions. We used four SN12C cell clones: SN12C (parent cell line), SN12C-clone 2, SN12C-clone 4 and SN12C-PM6 (3-5). The parental SN12C was established in culture from a primary renal cell carcinoma from a 43-year-old man as previously described (3). SN12C-clones 2 and 4 were isolated from SN12C by a double limiting dilution technique (7). SN12C-PM6 was established in vitro from a solitary metastatic lung HRCC nodule of $\mathrm{Balb} / \mathrm{c}$ nude mouse which had been injected with $1 \times 10^{6}$ viable parental SN12C into the renal subcapsule (5). SN12C-clone 2 and SN12C-PM6 have a higher metastatic potential than the SN12C $(4,5)$.

We also used three human scirrhous gastric cancer cell lines: OCUM-2M, OCUM-2D and OCUM-2MLN. OCUM-2M has a low metastatic potential, while OCUM-2D and OCUM-2MLN, which have a high metastatic potential, were previously established by Yashiro et al (8) and Fujihara et al (9). OCUM-2M, OCUM-2D and OCUM-2MLN were maintained in high-glucose Dulbecco's Modified Eagle's Medium (DMEM) with $10 \%$ fetal bovine serum (FBS) at $37^{\circ} \mathrm{C}$ in a $5 \% \mathrm{CO}_{2}$ atmosphere.

The SN12C cell clones were maintained in RPMI-1640 medium (Nissui, Tokyo, Japan) supplemented with $10 \%$ FBS at $37^{\circ} \mathrm{C}$ in a $5 \% \mathrm{CO}_{2}$ atmosphere.

We used SN12C clones and human scirrhous gastric cancer cell lines passaged less than fifteen times culture after the cells had been sent to our laboratory. We used passage number of 8th to 15th culture for 2-DE and western blot analysis.

As confirmation, we used seven cell lines with varying metastatic potential.

Sample preparation. Cells were homogenized in lysis buffer (50 mM Tris- $\mathrm{HCl}, \mathrm{pH} 7.5,165 \mathrm{mM}$ sodium chloride, $10 \mathrm{mM}$ sodium fluoride, $1 \mathrm{mM}$ sodium vanadate, $1 \mathrm{mM}$ PMSF, $10 \mathrm{mM}$ EDTA, $10 \mu \mathrm{g} / \mathrm{ml}$ aprotinin, $10 \mu \mathrm{g} / \mathrm{ml}$ leupeptin and $1 \% \mathrm{NP}-40$ ) on ice. The suspensions were incubated for $1 \mathrm{~h}$ at $4^{\circ} \mathrm{C}$, centrifuged at $21,500 \mathrm{xg}$ for $30 \mathrm{~min}$ at $4^{\circ} \mathrm{C}$, and the supernatants were stored at $-80^{\circ} \mathrm{C}$ until use (10-13).

Two-dimensional gel electrophoresis (2-DE). Eighty micrograms of protein were used for each 2-DE. For the first dimension, isoelectric focusing (IEF) was performed in an IPGphor 3 IEF unit (GE Healthcare, Buckinghamshire, UK) on $11 \mathrm{~cm}$, immobilized, $\mathrm{pH}$ 4.0-7.0 linear gradient strips (Bio-Rad, Hercules, CA, USA) at $20^{\circ} \mathrm{C}$ and $50 \mu \mathrm{A} /$ strip. Samples were mixed with $200 \mu \mathrm{l}$ of rehydration buffer $(8 \mathrm{M}$ urea, 2\% CHAPS, 0.01\% bromophenol blue, 1.2\% Destreak reagent and $0.5 \%$ IPG buffer; GE Healthcare) and loaded onto the IPGphor strip holder. IEF was performed using the following voltage program: rehydration $10 \mathrm{~h}$ (no voltage), IEF stepwise increase from 0 to $500 \mathrm{~V}$ for $240 \mathrm{~min}, 500-1,000 \mathrm{~V}$ for $60 \mathrm{~min}, 1,000-8,000 \mathrm{~V}$ for $240 \mathrm{~min}$, a linear increase from $8,000 \mathrm{~V}$ for $20 \mathrm{~min}$ and a final phase of $500 \mathrm{~V}$ from 20,000 to $30,000 \mathrm{Vh}$. In the second dimension, SDS-PAGE was performed on a precast polyacrylamide gel with a linear concentration gradient of 5-20\% (Bio-Rad), run at $200 \mathrm{~V}$ (14).

Flamingo staining. Agitation was carried out at all stages. After 2-DE, the gels were subjected to a fixing solution with $40 \%$ ethanol and $10 \%$ acetic acid for $4 \mathrm{~h}$. The gels were stained with Flamingo ${ }^{\mathrm{TM}}$ (Bio-Rad) overnight. Next, background stain was removed by wash solution containing $0.1 \%$ Tween-20 for $10 \mathrm{~min}$. Prior to imaging, the gels were washed twice in ultra pure water for $10 \mathrm{~min}$.

Image analysis and spot picking. The positions of protein spots on each 2-DE gel were recorded by using a Pro Express 2D Proteomic Imaging System (PerkinElmer, Waltham, MA, USA). Expression levels of the proteins were quantified by analyzing the intensity of each spot with Progenesis PG240 software (PerkinElmer). We selected the protein spots whose expression level significantly increased or decreased by $>1.5$-fold $(\mathrm{P}<0.05)$ compared with that of the $\mathrm{SN} 12 \mathrm{C}$ parent cell line in all experiments. In addition, these protein spots were picked up and analyzed further for correlation with the metastatic potential of SN12C clones as suggested from the results of 2-DE. The intensity of each spot was statistically analyzed by the Student's t-test. We performed five runs of 2-DE for image analysis.

Following statistical analysis, the gels were re-stained with See Pico ${ }^{\mathrm{TM}}$ (Benebiosis Co., Ltd., Seoul, Korea) and the selected spots were petal picked for mass spectrometry (MS) analysis. The spots, whose expression was significantly different between SN12C and SN12C-clone 2, SN12C-clone 4 or SN12-PM6, were selected for picking (15).

In-gel digestion. The See Pico dye was removed from the gel piece by rinsing two times in $60 \%$ methanol, $50 \mathrm{mM}$ ammonium bicarbonate and $5 \mathrm{mM}$ DTT for $10 \mathrm{~min}$. The protein sample in the gel piece was reduced twice in 50\% ACN, $50 \mathrm{mM}$ ammonium bicarbonate and $5 \mathrm{mM}$ DTT for $10 \mathrm{~min}$. The gel piece was dehydrated in 100\% ACN twice for $30 \mathrm{~min}$ and then rehydrated with an in-gel digestion reagent containing $10 \mu \mathrm{g} / \mathrm{ml}$ Sequencing Grade Modified Trypsin (Promega, Madison, WI, USA) in 30\% ACN, $50 \mathrm{mM}$ ammonium bicarbonate and $5 \mathrm{mM}$ DTT. This procedure for the in-gel digestion was performed overnight at $30^{\circ} \mathrm{C}$. The samples were lyophilized overnight using a Labconco Lyph-lock 1L Model 77400 (Labconco, Kansas, MO, USA). Lyophilized samples were dissolved in $0.1 \%$ formic acid.

Liquid chromatography and mass spectrometry (LC-MS). Samples dissolved in $0.1 \%$ formic acid were centrifuged at $21,500 \mathrm{x}$ g for $5 \mathrm{~min}$ and the supernatant was stored at $-80^{\circ} \mathrm{C}$ until use. An Agilent 1100 LC/MSD Trap XCT (Agilent Technologies, Palo Alto, CA, USA) was used for HPLC and MS/MS. Protein identification and post-transmodification search were performed in the Agilent Spectrum MILL MS Proteomics Workbench against the Swiss-Prot protein database search engine (http://kr.expasy.org/sprot/). Using the Agilent Spectrum MILL MS Proteomics Workbench, we performed the final assessment for protein identification using the peptide sequencing tag. The criteria for positive identification of proteins were set as follows: filter results by 
A

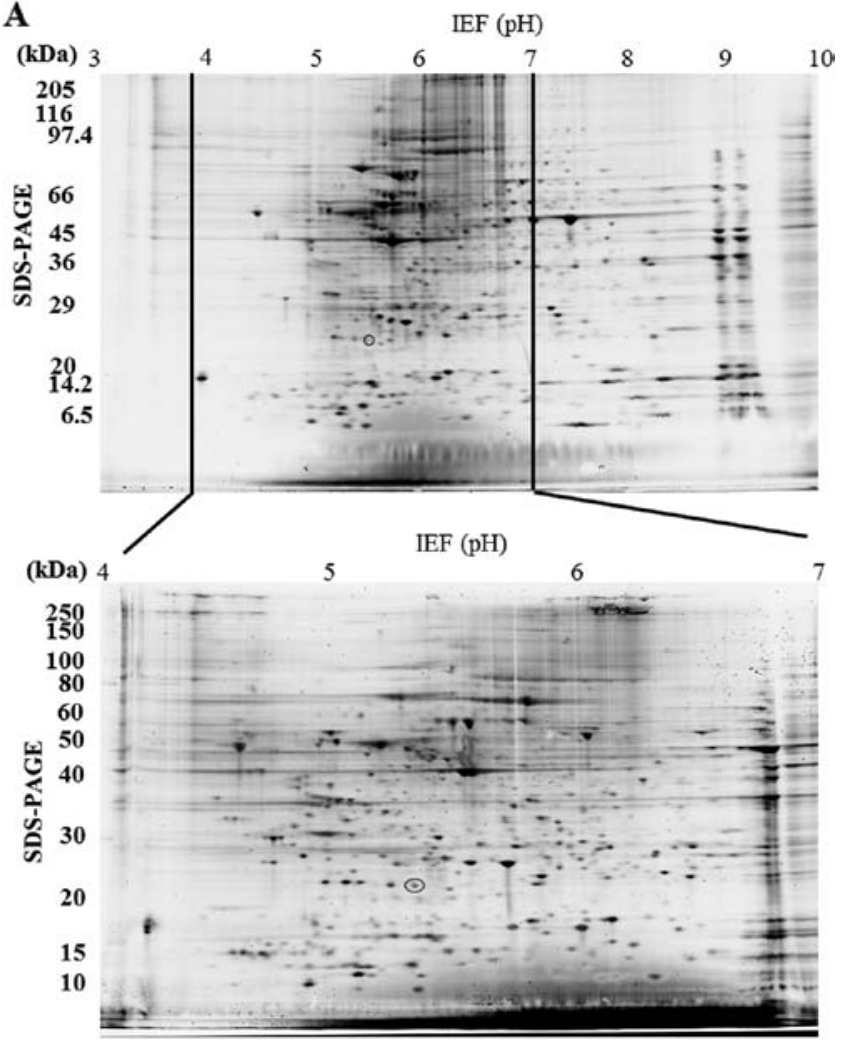

$\mathbf{B}$
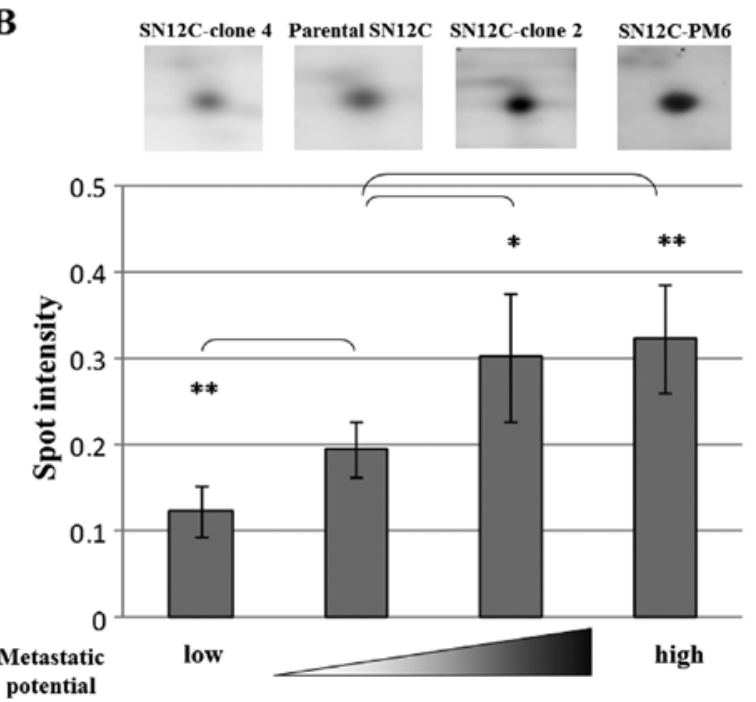

Figure 1. Two-dimensional electrophoresis (2-DE) pattern of parental SN12C when stained with Flamingo stain. (A) A total amount of $80 \mu \mathrm{g}$ was applied to each gel. The protein spot [glyoxalase 1 (GLO1)] whose expression levels varied with increasing metastatic potential in SN12C clones is indicated by a circle. The upper panel shows the pattern of 2-DE using a wide range of $\mathrm{pH} 3.0-10.0$. The lower panel shows the pattern of 2-DE using a narrow range of $\mathrm{pH} 4.0-7.0$. (B) Representative zoomed in sections of the circled segment in the overview gel for the SN12C clones SN12C-clone 4, SN12C-clone 2 and SN12C-PM6 as well as for the corresponding parental SN12C. The spots were significantly altered according to the metastatic potential of the SN12C clones. The graph shows the spot intensity from 2-DE. SN12C clones were statistically analyzed by the Student's t-test $\left({ }^{*} \mathrm{P}<0.05,{ }^{* *} \mathrm{P}<0.01\right)$. Results are expressed as the mean $\pm \mathrm{SD}(\mathrm{n}=5)$.

valid, filter by protein score 10.0 and filter peptide by score $>8, \%$ scored peak intensity $(\%$ SPI $)>70$. The Spectrum Mill workbench can search MS/MS spectra using an MS/MS ion search $(16,17)$.

Western blot analysis. Thirty and forty micrograms or forty micrograms of protein were used for 1-D or 2-D (IEF followed by SDS-PAGE) western blotting, respectively. After electrophoresis, proteins were transferred electrophoretically onto PVDF membranes (Immobilon-P; Millipore, Bedford, MA, USA) and blocked overnight at $4^{\circ} \mathrm{C}$ with Dulbecco's PBS (-) containing $5 \%$ skimmed milk and $0.2 \%$ Tween-20. The primary antibody used was anti-GLO1 mouse polyclonal antibody (dilution range 1:2,000; Abnova Corp., Taipei, Taiwan) and anti-actin goat polyclonal antibody (1:200) (dilution range 1:200; Santa Cruz Biotechnology, Inc., Santa Cruz, CA, USA). Membranes were incubated with the primary antibody for $1.5 \mathrm{~h}$ at room temperature, washed four times with Dulbecco's PBS (-) containing $0.2 \%$ Tween-20 and then incubated with a horseradish peroxidase-conjugated secondary antibody (dilution range 1:10,000; Jackson; ICN Biomedicals, Aurora, $\mathrm{OH}$, USA) for $1.5 \mathrm{~h}$ at room temperature. Membranes were developed using a chemiluminescense reagent (ECL Plus Western Blotting Detection Reagents; GE Healthcare). The immunoreactive protein bands were then quantified using Progenesis PG240 software (PerkinElmer). The differences in expression between parental SN12C and SN12C-clone 2, SN12C-clone 4 or SN12-PM 6, as well as between OCUM-2M and OCUM-2D or OCUM-2MLN, were analyzed statistically by the Student's t-test.

\section{Results}

Differential expression of GLO1 in 2-DE analysis. Protein expression in the $\mathrm{SN} 12 \mathrm{C}$ cell clones was assessed by 2-DE over a narrow $\mathrm{pH}$ range covering $\mathrm{pH}$ 4.0-7.0. This $\mathrm{pH}$ range was selected as our previous 2-DE study using a wide $\mathrm{pH}$ range covering $\mathrm{pH} 3.0-10.0$ showed that most spots were concentrated in the $\mathrm{pH} 4.0-7.0$ range (Fig. 1A).

The 2-DE was performed five times under the same conditions. From 200 to 350 spots were visualized on the 2-DE gels, and differences in spot intensity between parental SN12C and SN12C-clone 2, SN12C-clone 4 or SN12C-PM6 were analyzed with Progenesis PG240 software for each gel. We identified proteins whose expression was significantly different in agreement with the metastatic potential of the clone. Since several studies used the criteria of $>1.5$ fold-change, we used this criteria as well $(6,18,19)$. The spot whose expression level increased or decreased by $>1.5$-fold $(\mathrm{P}<0.05)$ are indicated by the circle (Fig. 1$)$. We found only one protein spot whose intensity was $>1.5$-fold different. The spot was consistent with our previous study (6) and significantly increased in high metastatic clones. However, this spot could not be identified by using MS analysis in our 
Table I. Identification of the protein which was differentially expressed between parental SN12C and other SN12C cell clones.

Low metastatic High metastatic clone clones

Accession no. Theoretical $\mathrm{pI}$ Theoretical Mr Protein name peptinct Sequence MS/MS Clone $4 \quad \overline{\text { Clone } 2 \quad \text { PM6 }}$

\begin{tabular}{llllllll}
\hline Q04760 & 5.12 & 20777.8 & Glyoxalase 1 & 4 & $25 \%$ & 53.49 & -
\end{tabular}

The protein spot was identified to be GLO1 by LC-MS/MS as described in Materials and methods. The spot intensity significantly decreased in the low metastatic clone and increased in high metastatic clones compared with parental SN12C by $>1.5$-fold $(\mathrm{P}<0.05)$; +, increased; -, decreased. MS, mass spectrometry; GLO1, glyoxalase 1; LC-MS/MS, liquid chromatography-tandem mass spectrometry.
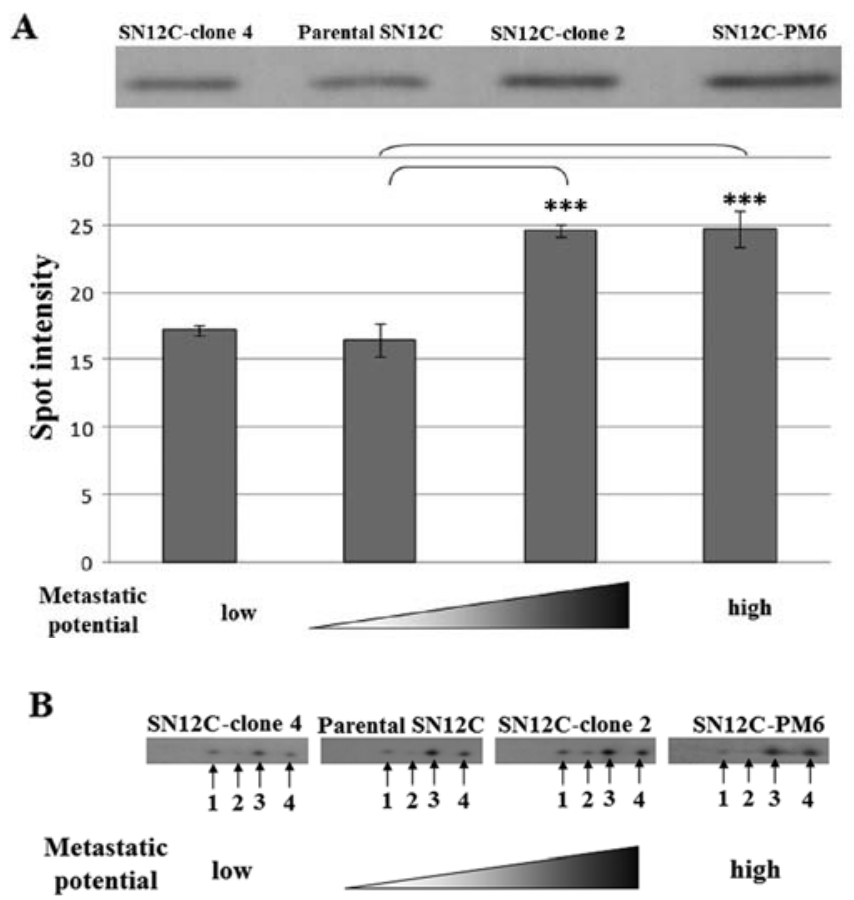

C

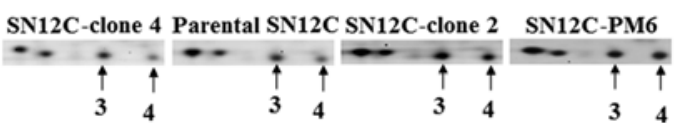

Metastatic potential low

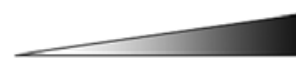

high

Figure 2. Western blot analysis for glyoxalase 1 (GLO1) in SN12C cell clones (A) Thirty micrograms of protein were used for each gel of SDS-PAGE. GLO1 was significantly increased in high metastatic clones. The graph shows the spot intensity of each band. The immunoreactive GLO1 band intensity between the clones was statistically analyzed by the Student's t-test $\left({ }^{* * * *} \mathrm{P}<0.001\right)$. Results are shown as the mean $\pm \mathrm{SD}(\mathrm{n}=6)$. (B) Forty micrograms of protein were used for 2-D western blotting as indicated in Materials and methods. (C) GLO1 protein spots on 2-DE gels were stained by Flamingo staining as described in Materials and methods.

previous study. In the present study, the protein spot showed lower expression in the low metastatic clone than parental SN12C and higher expression in the high metastatic clones. The spot with differential expression was picked, digested and used for MS analysis. The results of LC-MS/MS for this spot are shown in Table I.

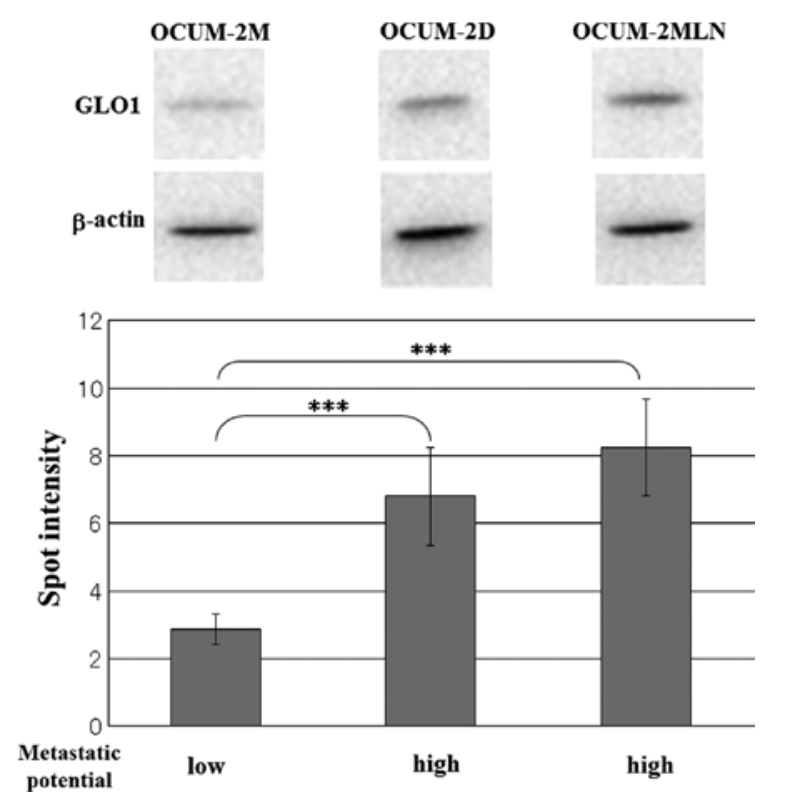

Figure 3. Western blot analysis for glyoxalase 1 (GLO1) in scirrhous gastric cancer cell lines. Protein $(30 \mu \mathrm{g})$ was used for each gel. GLO1 expression level in OCUM-2D and OCUM-2MLN was significantly increased compared to OCUM-2M. The graph shows spot intensity of each band. GLO1 expression level between the cell lines was statistically analyzed by the Student's t-test $\left({ }^{* * *} \mathrm{P}<0.01\right)$. Results are shown as the mean $\pm \operatorname{SD}(\mathrm{n}=5)$.

Increase of GLOI by western blotting in high metastatic potential SN12C clones. Western blot analysis of GLO1 was performed for all four SN12C clones. The immunoreactive GLO1 band was increased in the two high metastatic clones (Fig. 2A). In the 2-D immunoblotting for GLO1, four isoforms were observed for the SN12C clones (Fig. 2B); only Spots 3 and 4 (Fig. 2B and C) were identified as GLO1 from 2-DE analysis by using LC-MS/MS. However, the nature of the modifications of these GLO1 variants is thus far unknown. The intensity of Spot 4 (Figs. 1B and 2C) was significantly different in the SN12C clones compared with parental SN12C on 2-DE (Figs. 1B and 2C). Western blotting for Spot 4 in SN12C-clone 2, SN12C-clone 4 and SN12C-PM6 was consistent with 2-DE results.

To confirm whether an increase of GLO1 was also present in other cell lines observable of known differential metastatic potential, we performed western blotting for GLO1 in the scirrhous gastric cancer cell lines OCUM-2M, OCUM-2D and OCUM-2MLN. The results are shown in Fig. 3. The expression of GLO1 in the high metastatic scirrhous gastric cancer cell lines was also increased. 


\section{Discussion}

In the present study, we showed proteomic differential display analysis of SN12C HRCC cell clones having different metastatic potential by using 2-DE in the range of $\mathrm{pH} 4.0-7.0$, since most protein spots in our previous study were separated in the range of $\mathrm{pH} 4.0-7.0$ on the gels by using 2-DE in the range of $\mathrm{pH}$ 3.0-10.0 (6). Only one protein spot level was found to be significantly different in accordance with the metastatic potential of the cells. The protein spot was identified as GLO1 by LC-MS/MS. Western blotting with a specific antibody against GLO1 confirmed an increase in GLO1 expression in high metastatic cells compared with low metastatic cells. By 2-D immunoblotting for GLO1, four isoforms were observed in SN12C clones. Spot 4 in SN12C-clone 2, SN12C-clone 4 and SN12C-PM6 were consistent with the significantly different spot observed in the 2-DE results. Furthermore, western blotting for GLO1 in scirrhous gastric cancer cell lines was performed. GLO1 expression was increased in the high metastatic OCUM cell line.

GLO1 is part of the glyoxalase system in the cytosol of cells, a side-product of glycolysis. This system is one of the most effective detoxification systems for reactive $\alpha$-oxoaldehydes, including methylglyoxal (MG) (20). The major physiological substrate for GLO1 is MG. GLO1 plays an important role in catalyzing the conversion of MG to S-D-lactoylglutathione. In addition, GLO1 activity in situ is proportional to the cellular concentration of glutathione $(20,21)$. Several reports suggested that different isoforms of GLO1 exist (22-25) and these isoforms are subjected to several post-translational modifications, including a nitric-oxide (NO)-responsive form and phosphorylation (23). NO-responsive GLO1 appeared to be the most basic isoform of GLO1 on 2-DE gels $(22,23)$. In the present study, the most basic isoform of GLO1 was significantly altered in the SN12C clones. However, the nature of these modifications present in the additional GLO1 variants is thus far unknown. The GLO1 protein may be modified post-translationally. Previous reports indicated that a correlation between the expression level of GLO1 and the nature of various tumors exists (26-29). Cheng et al (26) reported that high expression of GLO1 in gastric cancer enhances the metastatic ability of tumor cells in vitro and in vivo. MG has long been regarded as a natural anticancer agent and, therefore, GLO1 inhibitors have long been considered possible anticancer agents $(30,31)$. For example, Santel et al (30) reported curucumin (a naturel phenol) inhibits GLO1 and has a strong impact on highly proliferative and invasive tumor cells such as $1321 \mathrm{~N}, \mathrm{PC}-3$ and MDA-MB-231. Recently, GLO1 was identified as a methyl-gerfelin (M-GFN)-binding protein. M-GFN (a methyl benzoate) inhibited the enzymatic activity of GLO1 in vitro and in situ (32). Imoto (33) reported that invasion of cancer cells was inhibited by the inhibitory activity of M-GFN. These results suggest that the increased GLO1 expression provides advantages to the metastatic potential in SN12C clones. In addition, inhibition of GLO1 may have therapeutic potential for the treatment of metastatic cancer.

The increase of GLO1 and the decrease of UCH-L1 are significant at the protein level in high metastatic clones compared with parental SN12C. Our findings strongly suggest that GLO1 and UCH-L1 are associated with the metastatic potential of SN12C HRCC cells. However, the type of relationship that exists between increased GLO1 and decreased UCH-L1 remains to be clarified.

Collectively, in the present study, we performed differential display analysis of proteins in an attempt to find candidate proteins linked to the metastatic potential of $\mathrm{SN} 12 \mathrm{C}$ renal cell carcinoma clones by using 2-DE in the narrow range of $\mathrm{pH}$ 4.0-7.0. From 200 to 350 spots were visualized on the 2-DE gels, but only one GLO1 spot was significantly increased in the high metastatic clones and decreased in the low metastatic clone compared with parental SN12C. The increase of GLO1 was confirmed in high metastatic SN12C clones and additionally in metastatic scirrhous gastric cancer cell lines. Further studies are required to clarify the biological significance of GLO1 in the metastatic potential of these cell lines. The type of post-translational modification that occurred in the GLO1 isoforms and the difference between these isoforms need to be examined. In addition, we need to assess the correlation between GLO1 and other metastasis-related factors, including UCH-L1.

\section{References}

1. Geiger TR and Peeper DS: Metastasis mechanisms. Biochim Biophys Acta 1796: 293-308, 2009.

2. Fidler IJ: Models for spontaneous metastasis. Cancer Res 66: 9787, 2006.

3. Naito S, von Eschenbach AC, Giavazzi R and Fidler IJ: Growth and metastasis of tumor cells isolated from a human renal cell carcinoma implanted into different organs of nude mice. Cancer Res 46: 4109-4115, 1986.

4. Naito S, Walker SM, von Eschenbach AC and Fidler IJ: Evidence for metastatic heterogeneity of human renal cell carcinoma. Anticancer Res 8: 1163-1167, 1988.

5. Naito S, Walker SM and Fidler IJ: In vivo selection of human renal cell carcinoma cells with high metastatic potential in nude mice. Clin Exp Metastasis 7: 381-389, 1989.

6. Tanaka T, Kuramitsu Y, Fujimoto M, Naito S, Oka M and Nakamura K: Downregulation of two isoforms of ubiquitin carboxyl-terminal hydrolase isozyme L1 correlates with high metastatic potentials of human SN12C renal cell carcinoma cell clones. Electolophoresis 29: 2651-2659, 2008.

7. Fidler IJ and Kripke ML: Metastasis results from preexisting variant cells within a malignant tumor. Science 197: 893-895, 1977.

8. Yashiro M, Chung YS, Nishimura S, Inoue T and Sowa M: Peritoneal metastatic model for human scirrhous gastric carcinoma in nude mice. Clin Exp Metastasis 14: 43-54, 1996.

9. Fujihara T, Sawada T, Hirakawa K, Chung YS, Yashiro M, Inoue $\mathrm{T}$ and Sowa M: Establishment of lymph node metastatic model for human gastric cancer in nude mice and analysis of factors associated with metastasis. Clin Exp Metastasis 16: 389-398, 1998.

10. Fukagawa Y, Nishikawa J, Kuramitsu Y, Iwakiri D, Takada K, Imai S, Satake M, et al: Epstein-Barr virus upregulates phosphorylated heat shock protein $27 \mathrm{kDa}$ in carcinoma cells using the phosphoinositide 3-kinase/Akt pathway. Electrophoresis 29: 3192-3200, 2008.

11. Mori-Iwamoto S, Kuramitsu Y, Ryozawa S, Taba K, Fujimoto M, Okita K, Nakamura K and Sakaida I: A proteomic profiling of gemcitabine resistance in pancreatic cancer cell lines. Mol Med Rep 1: 429-434, 2008.

12. Mori-Iwamoto S, Kuramitsu Y, Ryozawa S, Mikuria K, Fujimoto M, Maehara S, Maehara Y, et al: Proteomics finding heat shock protein 27 as a biomarker for resistance of pancreatic cancer cells to gemcitabine. Int J Oncol 31: 1345-1350, 2007.

13. Hayashi E, Kuramitsu Y, Okada F, Fujimoto M, Zhang X, Kobayashi M, Iizuka N, et al: Proteomic profiling for cancer progression: differential display analysis for the expression of intracellular proteins between regressive and progressive cancer cell lines. Proteomics 5: 1024-1032, 2005. 
14. Kuramitsu Y, Miyamoto H, Tanaka T, Zhang X, Fujimoto M, Ueda K, Tanaka T, et al: Proteomic differential display analysis identified upregulated astrocytic phosphoprotein PEA-15 in human malignant pleural methothelioma cell lines. Proteomics 9: 5078-5089, 2009 .

15. Tamesa M, Kuramitsu Y, Fujimoto M, Maeda N, Nagashima Y, Tanaka T, Yamamoto S, et al: Detection of autoantibodies against cyclophilin A and triosephosphate isomerase in sera from breast cancer patients by proteomic analysis. Electrophoresis 30 : 2168-2181, 2009

16. Takashima M, Kuramitsu Y, Yokoyama Y, Iizuka N, Harada T, Fujimoto M, Sakaida I, et al: Proteomic analysis of autoantibodies in patients with hepatocellular carcinoma. Proteomics 6 : 3894-3900, 2006.

17. Takashima M, Kuramitsu Y, Yokoyama Y, Iizuka N, Fujimoto M, Nishisaka T, Okita K, Oka M and Nakamura K: Overexpression of alpha enolase in hepatitis $\mathrm{C}$ virus-related hepatocellular carcinoma: association with tumor progression as determined by proteomic analysis. Proteomics 5: 1686-1692, 2005.

18. Ding Q, Wu Z, Guo Y, Zhao C, Jia Y, Kong F, Chen B, et al: Proteome analysis of up-regulated proteins in the rat spinal cord induced by transection injury. Proteomics 6: 505-518, 2006.

19. Henschke P, Vorum H, Honoré B and Rice GE: Protein profiling the effects of in vitro hyperoxic exposure on fetal rabbit lung. Proteomics 6: 1957-1962, 2006.

20. Shinohara M, Thornalley PJ, Giardino I, Beisswenger P, Thorpe SR, Onorato J and Brownlee M: Overexpression of glyoxalase-I in bovine endothelial cells inhibits intracellular advanced glycation endproduct formation and prevents hyperglycemia-induced increases in macromolecular endocytosis. J Clin Invest 101: 1142-1147, 1998.

21. Thornalley PJ: Glyoxalase I - structure, function and a critical role in the enzymatic defence against glycation. Biochem Soc Trans 31: 1343-1348, 2003.

22. Mitsumoto A, Kim KR, Oshima G, Kunimoto M, Okawa K, Iwamatsu A and Nakagawa Y: Glyoxalase I is a novel nitric oxide responsive protein. Biochem J 3: 837-844, 1999.

23. de Hemptinne V, Rondas D, Vandekerckhove $J$ and Vancompernolle K: Tumour necrosis factor induces phosphorylation primarily of the nitric-oxide-responsive form of glyoxalase I. Biochem J 407: 121-128, 2007.
24. de Hemptinne V, Rondas D, Toepoel M and Vancompernolle K: Phosphorylation on Thr-106 and NO-modification of glyoxalase I suppress the TNF-induced transcriptional activity of NF-kappaB. Mol Cell Biochem 325: 169-178, 2009.

25. Van Herreweghe F, Mao J, Chaplen FW, Grooten J, Gevaert K, Vandekerckhove $\mathrm{J}$ and Vancompernolle K: Tumor necrosis factor-induced modulation of glyoxalase I activities through phosphorylation by PKA results in cell death and is accompanied by the formation of a specific methylglyoxal-derived AGE. Proc Natl Acad Sci USA 99: 949-954, 2002.

26. Cheng WL, Tsai MM, Tsai CY, Huang YH, Chen CY, Chi HC, et al: Glyoxalase-I is a novel prognosis factor associated with gastric cancer progression. PLoS One 7: e34352, 2012.

27. Wang Y, Kuramitsu Y, Ueno T, Suzuki N, Yoshino S, Izuka N, Akada J, et al: Glyoxalase I (GLO1) is up-regulated in pancreatic cancerous tissues compared with related non-cancerous tissues. Anticancer Res 32: 3219-3222, 2012.

28. Fonseca-Sánchez MA, Rodríguez Cuevas S, MendozaHernández G, Bautista-Piña V, Arechaga Ocampo E, Hidalgo Miranda A, Quintanar Jurado V, et al: Breast cancer proteomics reveals a positive correlation between glyoxalase 1 expression and high tumor grade. Int J Oncol 41: 670-680, 2012.

29. Jones MB, Krutzsch H, Shu H, Zhao Y, Liotta LA, Kohn EC and Petricoin EF III: Proteomic analysis and identification of new biomarkers and therapeutic targets for invasive ovarian cancer. Proteomics 2: 76-84, 2002.

30. Santel T, Pflug G, Hemdan NY, Schäfer A, Hollenbach M, Buchold M, Hintersdorf A, et al: Curcumin inhibits glyoxalase 1: a possible link to its anti-inflammatory and anti-tumor activity. PLoS One 3: e3508, 2008.

31. Tsuruo T, Naito M, Tomida A, Fujita N, Mashima T, Sakamoto H and Haga N: Molecular targeting therapy of cancer: drug resistance, apoptosis and survival signal. Cancer Sci 94: 15-21, 2003.

32. Kawatani M, Okumura H, Honda K, Kanoh N, Muroi M, Dohmae N, Takami M, et al: The identification of an osteoclastogenesis inhibitor through the inhibition of glyoxalase I. Proc Natl Acad Sci USA 105: 11691-11696, 2008.

33. Imoto M: Isolation of novel GGPP synthase-inhibitor Gerfelin and evaluation of its activity. Bunshi, Chobunshi, Chokozotai Rikogaku Heisei 15 Nendo Kenkyu Seika Hokokusho Gakujutsu Furonthia, pp597-656, 2004 (In Japanese). 